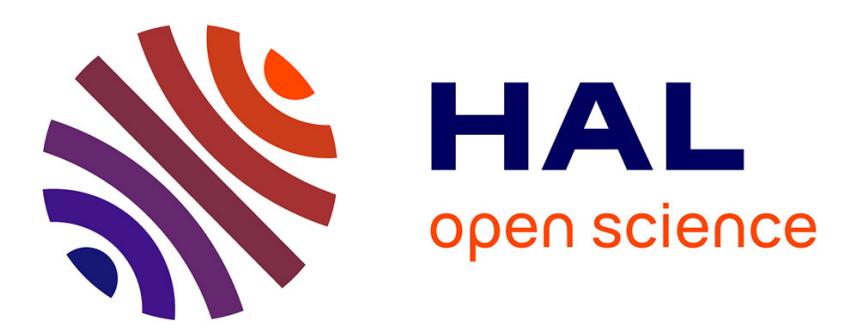

\title{
On the potential of ultrasound elastography for pressure ulcer early detection.
}

Jean-François Deprez, Elisabeth Brusseau, Jérémie Fromageau, Guy Cloutier, Olivier Basset

\section{- To cite this version: \\ Jean-François Deprez, Elisabeth Brusseau, Jérémie Fromageau, Guy Cloutier, Olivier Basset. On the potential of ultrasound elastography for pressure ulcer early detection.. Medical Physics, 2011, 38 (4), pp.1943-50. 10.1118/1.3560421 . inserm-00598688}

\section{HAL Id: inserm-00598688 https://www.hal.inserm.fr/inserm-00598688}

Submitted on 7 Jun 2011

HAL is a multi-disciplinary open access archive for the deposit and dissemination of scientific research documents, whether they are published or not. The documents may come from teaching and research institutions in France or abroad, or from public or private research centers.
L'archive ouverte pluridisciplinaire HAL, est destinée au dépôt et à la diffusion de documents scientifiques de niveau recherche, publiés ou non, émanant des établissements d'enseignement et de recherche français ou étrangers, des laboratoires publics ou privés. 


\title{
${ }^{1}$ On the potential of ultrasound elastography for pressure ulcer 2 early detection
}

\author{
3 Jean-François Deprez ${ }^{\mathrm{a})}$ and Elisabeth Brusseau \\ 4 CREATIS Laboratory, Université de Lyon, Insa de Lyon, Université Lyon 1, CNRS UMR5220, \\ $5 \quad$ Inserm U1044, Villeurbanne 69621, France \\ $6 \quad$ Jérémie Fromageau and Guy Cloutier \\ $7 \quad$ Laboratory of Biorheology and Medical Ultrasonics, University of Montreal Hospital Research Center \\ 8 (CRCHUM), Montreal, Québec H2L 2W5, Canada \\ Olivier Basset \\ CREATIS Laboratory, Université de Lyon, Insa de Lyon, Université Lyon 1, CNRS UMR5220, \\ Inserm U1044, Villeurbanne 69621, France
}

(Received 19 October 2010; revised 25 January 2011; accepted for publication 6 February 2011; published $\mathrm{xx}$ xx $\mathrm{xxxx}$ )

Purpose: Pressure ulcers are areas of soft tissue breakdown induced by a sustained mechanical stress that damages the skin and underlying tissues. They represent a considerable burden to the society in terms of health care and cost. Yet, techniques for prevention and detection of pressure ulcers still remain very limited. In this article, the authors investigated the potential of ultrasound elastography for pressure ulcer early detection. Elastography is an imaging technique providing local information on biological tissue mechanical properties. It is relevant for pressure ulcer detection as this pathology is associated with a gradual stiffening of damaged tissues, beginning in the deeper tissues and progressing toward the skin surface.

Methods: A 2D ultrasound elastography method was proposed and its ability in terms of pressure ulcer detection was validated through numerical simulations and physical acquisitions on pressure ulcer mimicking phantoms. In vivo experiments on a rat model are also reported. A maintained pressure was applied on the animal thigh, with a view to generate a pressure ulcer, and ultrasound data were acquired and processed before and after application of this pressure.

Results: Numerical simulations demonstrated that a pressure ulcer can theoretically be detected at a very early stage with ultrasound elastography. Even when the ulcer region was characterized by a low stiffening (ratio of 1.8 relative to normal tissues), the corresponding elastogram clearly underlined the pathological area. This observation was confirmed by the results obtained on a physical phantom mimicking a pressure ulcer at an early stage. Computed elastograms showed strain differences between areas mimicking healthy and pathological tissues. Results corresponding to in vivo experiments revealed a difference in the way tissues behaved before and after the pressure was applied on the animal thigh, which strongly suggests the presence of a pathological area.

Conclusions: Experiments demonstrated that ultrasound elastography is a promising technique for pressure ulcer detection, especially at an early stage of the pathology, when the disease is still visually undetectable. In the absence of any gold standard method, this is also a first step toward the development of a quantitative technique. (C) 2011 American Association of Physicists in Medicine. [DOI: $10.1118 / 1.3560421]$

Key words: pressure ulcer, ultrasound, elastography, medical imaging

\section{I. INTRODUCTION}

42 Pressure ulcers, or bedsores, are areas of soft tissue break43 down induced by a sustained mechanical stress that damages 44 the skin and underlying tissues. They often appear after a 45 prolonged period of immobility, during which biological tis46 sues undergo a maintained pressure from a support (gener47 ally a bed or a wheelchair). Pressure ulcers mostly affect 48 people with sensitivity loss or limited mobility, who are un49 able to feel and/or release the pressure. Elderly people, pa50 tients in hospital recovery and persons with spinal cord in51 jury are especially at risk.

52 Few statistics are available to estimate the extent of this pathology. In 2001, wide variations were found for the $\mathbf{5 3}$ prevalence of pressure ulcer in the United States: ${ }^{1} 10 \%-18 \% 54$ in acute care, $2.3 \%-28 \%$ in long-term care, and $0 \%-29 \%$ in 55 home care. In the United Kingdom, prevalence was esti- 56 mated at $18 \%$ in $1993 .^{2}$ As life expectancy increases in west- $\mathbf{5 7}$ ern countries, this disease becomes a growing issue and rep- 58 resents a considerable burden to the society in terms of 59 health care and associated cost. ${ }^{3,4} \quad 60$

Despite these numbers, techniques for prevention and de- 61 tection of pressure ulcer still remain very limited. For in- 62 stance, risk scales do exist but they are mostly used for hos- 63 pitalized patients, and their efficiency is largely questioned. ${ }^{5} 64$ 
65 Thus, detection and prevention are mainly based on the ex66 perience of the health-care personnel.

67 Different approaches were developed to study skin 68 wounds. A good review of wound measurement techniques 69 can be found in Ref. 6. These techniques can be divided into 70 two categories: Visual assessment tools, based on color and 71 digital image analysis, and physiological-based tools (i.e., 72 blood flow, force, or pressure measurements). More recently, 73 Weber et al. $^{7}$ developed a device to map the electrical im74 pedance of a wound in order to determine the wound sever75 ity. Treuillet et al. ${ }^{8}$ also presented an original approach to 76 build 3D models of skin wounds from digital camera snap77 shots, allowing quantitative measurements of the wound, 78 such as surface, depth, or volume.

79 Attempts to develop quantitative techniques for detection 80 or prevention of pressure ulcers are far less numerous. In 81 Ref. 9, 15 pressure ulcers were examined using four different 82 noninvasive techniques: Skin temperature, redness index, 83 skin retraction time, and ultrasound (US) scanning. Results 84 showed that temperature and retraction time could not alone 85 characterize the ulceration stage, while a redness index was 86 useful in some cases. Ultrasound image processing could de87 tect a subepidermal layer, which may be a good indicator that 88 the skin is at risk. One can finally cite Ref. 10, where pre89 vention of pressure ulcer was based on both movement mea90 surement and autonomous nervous system analysis. Even 91 though these studies were constructive, it is still relevant to 92 develop new quantitative approaches.

93 This lack of quantitative assessment tools is likely the 94 result of a limited understanding of the pathology itself. 95 There is indeed very little literature on the process that trig96 gers the formation of pressure ulcers.

97 To date, the most established theory is that tissue com98 pression locally results in the occlusion of blood 99 capillaries. ${ }^{11-13}$ The mechanism leading to the formation of a 100 pressure ulcer is linked with a shortage of blood supply, 101 called ischemia, which prevents the natural exchanges of 102 oxygen and nutrients. If the microcirculatory flow distur103 bance remains for too long, the ischemic condition leads to 104 cell death and tissue damages.

105 It is also acknowledged that pressure ulcers are associated 106 with a stiffening of damaged tissues, as shown in Refs. 14 107 and 15 on a rat model and on a realistic numerical model of 108 human buttocks, respectively.

109 Finally, recent studies suggest that pressure ulcers can 110 form either superficially or within deep tissues. ${ }^{11,16,17}$ Super111 ficial pressure ulcers arise in the skin. They are mainly 112 caused by shear stresses and are easily detected by careful 113 skin inspection. On the other hand, deep pressure ulcers (also 114 called deep tissue injuries) develop in deep muscle layers 115 covering bony prominences and are caused by sustained 116 compression of the tissues. They follow a "deep-to117 superficial" pattern and progress toward the skin surface. 118 Therefore, severe necrosis of subcutaneous tissues may occur 119 while the skin shows only minor signs of tissue breakdown. 120 Consequently, deep ulcers are very difficult to identify. Since 121 deep pressure ulcers are characterized by stiffening and a deep-to-superficial growing process, providing information 122 on tissue local mechanical properties could be of major in- 123 terest for physicians.

Accordingly, US elastography is proposed for pressure ul- 125 cer early detection. US elastography ${ }^{18,19}$ is indeed providing 126 to clinicians information on the local mechanical properties 127 of soft biological tissues. This information, generally dis- 128 played as an image, enables the detection of unexpected stiff 129 area within healthy tissues. Typical applications of US elas- 130 tography are targeted to diseases such as breast and prostate 131 cancer, ${ }^{20,21}$ liver fibrosis, ${ }^{22}$ and atherosclerosis. ${ }^{23} \quad 132$

Potentially, pressure ulcer US elastography could be used 133 for diagnosis and wound healing follow-up. US elastography 134 also has the advantage of being noninvasive, low-cost, and 135 portable.

136

In this paper, we investigated the potential of quasistatic 137 US elastography for pressure ulcer early detection. The paper 138 is organized as follows. Section II provides a description of 139 the US elastography method, followed by its application to 140 numerical data in Sec. III, and to a pressure ulcer mimicking 141 phantom in Sec. IV. Section V is dedicated to the presenta- 142 tion of results obtained during an in vivo experiment. The 143 final section provides a critical discussion and concluding 144 remarks.

\section{METHOD}

146

Depending on the mechanical excitation applied to the 147 tissues, there are mainly two approaches in US elastography, 148 termed quasistatic elastography ${ }^{19,24-26}$ and dynamic 149 elastography. ${ }^{18,27-29}$ This paper falls into the category of qua- 150 sistatic elastography, which investigates tissue deformation 151 under compression. In quasistatic elastography, information 152 on tissue local mechanical properties is deduced from a 153 simple principle: Subjected to a given stress, soft areas will 154 deform more than stiffer ones. In practical terms, for a tissue 155 under investigation, two US radiofrequency (RF) data sets 156 are acquired, corresponding to two different stress levels 157 called pre- and postcompression states. Pattern changes in- 158 duced by the stress within the RF signals are then analyzed 159 to compute a map of local strains.

160

The different steps leading to this local strain estimation 161 are briefly explained hereafter to make the paper self- 162 contained. The interested reader can refer to Refs. 26 and 30163 for more details.

\section{II.A. Image deformation model}

To achieve a local estimation, images were divided into 166 multiple small regions-of-interest (ROIs) and for each ROI, 167 the effects of the tissue compression were analyzed. Most of 168 the 2D techniques used in elastography model the 169 compression-induced tissue motion as a local 2D translation 170 and then compute strain as the gradient of the resulting dis- 171 placement field. Contrary to these techniques, we considered 172 that the effect of compression on each ROI can be modeled 173 as an affine transformation. In addition to a 2D translation, a 174 scaling of the ROI was also considered, which reflects more 175 realistically the tissue deformation at this location. 


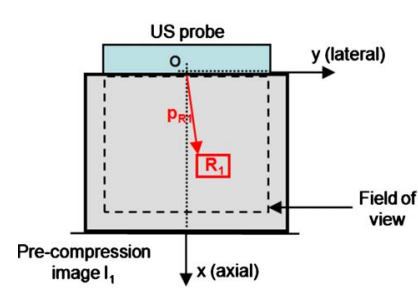

(a)

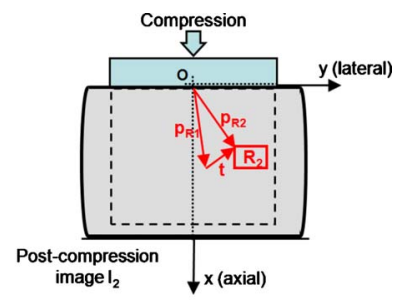

(b)
FIG. 1. Region of interest positioning in (a) the precompression image and (b) the postcompression image.

177 However, US data resolution is highly anisotropic. Owing 178 to US device characteristics, lateral resolution is much 179 coarser than the axial resolution, which makes an accurate 180 estimation of a scaling factor difficult in the lateral direction. 181 This has led us to consider only an axial scaling of the ROI, 182 the lateral deformation being taken into account in the lateral 183 translation of the ROI.

\section{II.B. ROI displacement}

185 Let us denote $I_{1}$ and $I_{2}$ the pre- and postcompression im186 ages, respectively. Let us consider $R_{1}$ a ROI of dimensions $187 L_{x 1} \times L_{y 1}$, located at $p_{R 1}$ in $I_{1}$. Finally, let us denote $R_{2}$ the $188 \mathrm{ROI}$ in $I_{2}$ corresponding to the same tissue region after de189 formation. Its axial and lateral dimensions $L_{x 2}$ and $L_{y 2}$ are 190 such that $L_{x 2}=\alpha \cdot L_{x 1}$ (axial scaling) and $L_{y 2}=L_{y 1}$. The posi191 tion of $R_{2}$ in $I_{2}$, namely, $p_{R 2}$, is also different from $p_{R 1}$, as 192 shown in Fig. 1. We therefore initialize $R_{2}$ at its most prob193 able position in $I_{2}$ as

$194 \quad p_{R 2 \text { ini }}=p_{R 1}+t$,

195 with $t\left(t_{\mathrm{ax}}, t_{\mathrm{lat}}\right)$ a $2 \mathrm{D}$ translation. This translation vector repre196 sents the effects of tissue deformation on the position of $R_{2}$. 197 In particular, its axial component $t_{\mathrm{ax}}$ results from the axial 198 scaling of the regions located between the probe and the ROI 199 currently considered. It can therefore be entirely determined 200 from previous computations by beginning the estimation pro201 cess for the regions at the interface with the probe (where $202 t_{\mathrm{ax}}=0$ ) and propagating this process to regions downward. 203 Similarly, the lateral shift $t_{\text {lat }}$ is directly given by the previous 204 estimations, by beginning the estimation process for the re205 gions at the center of the probe and propagating this process 206 to the lateral sides.

\section{II.C. Problem formulation}

208 For the positions of $R_{1}$ and $R_{2}$ that have been determined, 209 two local parameters must now be estimated: An axial scal210 ing $\alpha$ and a residual lateral shift $\tau$. This estimation is per211 formed through the minimization of an objective function $f$, 212 which is defined as the opposite of the normalized correla213 tion coefficient between $R_{1}$ and $R_{2}, R_{2}$ varying with these 214 parameters.

215 Since RF US data are oscillating signals by nature, the 216 function $f$ may exhibit several local minima. To narrow the 217 optimization process around the area corresponding to the global minimum, the range of values allowed for the param- 218 eters is reduced. This reduction in the parameter domain is 219 reasonable since in elastography, deformations induced by 220 the external load are of small magnitude (a few \%). $R_{2}$ is 221 therefore sought in a small region immediately surrounding 222 its initial position. As a result, the optimization is subjected 223 to a set of linear inequality constraints on the parameters and 224 the problem to be solved can be formulated as

$$
\left[\begin{array}{ll}
\hat{\alpha} & \hat{\tau}
\end{array}\right]=\underset{\alpha, \tau}{\arg \min } f(\alpha, \tau) \text { with }\left\{\begin{array}{c}
\alpha_{\min } \leq \alpha \leq \alpha_{\max } \\
\tau_{\min } \leq \tau \leq \tau_{\max }
\end{array} .\right.
$$

Typically, axial strain is restricted to the range [0\%-6\%] and 227 lateral shift is limited to three times the RF line interspacing 228 (i.e., $\alpha_{\min }=0.94, \alpha_{\max }=1, \tau_{\min }=-3$, and $\tau_{\max }=3$ ).

In practical terms, this optimization process is imple- 230 mented, thanks to a sequential quadratic programming 231 methodology, ${ }^{31}$ together with an active set strategy. To en- 232 sure a reliable estimation and prevent the objective function 233 from being trapped in a local minimum, correction proce- 234 dures are also considered based on multiple initializations of 235 the process $^{26,30}$

\section{II.D. Axial strain computation}

In this paper, the mechanical parameter we are interested 238 in is the medium's axial strain. This information is directly 239 deduced from the estimated axial scaling factor $\hat{\alpha}$, according 240 to the following relation:

241

$$
\varepsilon=\hat{\alpha}-1 \text {. }
$$

It should be stressed that with Eq. (3), strain is negative for a 243 compression and positive for a dilatation. Because in this 244 study, all experiments were performed by applying a com- 245 pression to the observed media, computed strain values were 246 negative. For simplification purposes, we chose to display 247 the opposite of the strain, leading to elastograms with posi- 248 tive values, keeping in mind that they represent the percent- 249 age of medium compression.

\section{NUMERICAL SIMULATIONS}

Deep pressure ulcers develop at the deepest level and pro- 252 ceeds outward until they reach the skin. ${ }^{11,16,17}$ At an early 253 stage of the pathology, deep tissues are therefore damaged 254 while the epidermis is still intact. Besides, areas particularly 255 exposed to pressure ulcers are those over bony prominences, 256 such as the sacrum, hips, and heels, which represent $80 \%$ of 257 bedsores. ${ }^{32}$ From this background, a numerical phantom of 258 pressure ulcer was designed with regard to these observa- 259 tions, and the potential of US elastography for the early de- 260 tection of this condition was investigated.

261

The phantom of $30 \times 20 \times 4 \mathrm{~mm}^{3}$ modeled a deep ulcer 262 at the very beginning of the pathological process (Fig. 2). It 263 was made of three different regions:

- Healthy soft tissues in contact with the US probe, 265

- A bony prominence at the bottom, which is a privileged 266 configuration for pressure ulcer formation, 


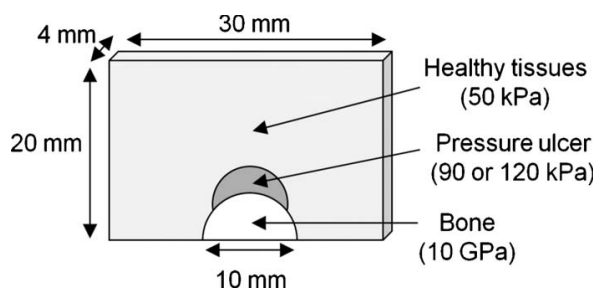

FIG. 2. Sketch of the numerical phantom mimicking a pressure ulcer at an early stage.

- And an intermediate layer mimicking the indevelopment ulcer, located above the bone, and whose limited size made it visually undetectable from the phantom upper surface.

272 The phantom mechanical properties were defined as fol273 lows: The Young's modulus of the bone was set to $10 \mathrm{GPa}$ 274 and that of healthy tissues was set to $50 \mathrm{kPa}$. These are 275 orders of magnitude reported in literature. ${ }^{33-35}$ The modeling 276 of the pressure ulcer was based on the following observation. 277 During their experiments, Gefen et al. ${ }^{14}$ measured a 1.8-3.3278 fold stiffening of tissues damaged by a pressure ulcer com279 pared to normal ones. Accordingly, two different values were 280 chosen for the Young's modulus of the pressure ulcer: Either $28190 \mathrm{kPa}$, corresponding to the lowest ratio $(1.8 \times 50 \mathrm{kPa})$, or $282120 \mathrm{kPa}$, corresponding to a middle range value. In addition, 283 a third configuration was considered for control. It repre284 sented a "healthy" state, with no pressure ulcer (Young's 285 modulus of $50 \mathrm{kPa}$ ).

286 To obtain the postcompression configuration, a $1.5 \mathrm{kPa}$ 287 stress was applied on the upper surface of phantoms down288 ward. The corresponding tissue deformations were computed 289 through the use of a finite element modeling (FEM) package 290 (COMSOL MULTIPHYSICS, Comsol Inc., USA).

291 From an acoustical point of view, tissues were modeled as 292 a large number of punctual scatterers randomly distributed in 293 the region-of-interest. In the absence of information related 294 to pressure ulcer echogenicity and since our technique is 295 based only on tissue's stiffness differences, healthy and 296 pathological tissues were considered as having identical 297 acoustical properties. Consequently, a standard B-mode im298 age did not allow seeing any difference between these two 299 regions. On the other hand, the bone was designed to be 300 hyperechoic compared to soft tissues.

301 US RF data were the result of the convolution between 302 scatterers and a point spread function simulating the acous303 tical response of the scanner. ${ }^{36}$ Simulations were performed 304 for both the pre- and the postcompression states, displace305 ments of scatterers between the two states being given by the 306 FEM.

307 The strain estimator described in Sec. II was applied to 308 these simulated data. The corresponding results, together 309 with theoretical fields, are given in Fig. 3. We can first ob310 serve that the estimated elastograms are close to the theoret311 ical fields provided by the FEM. They both exhibit similar 312 patterns of strains, which is satisfying regarding the strain 313 estimation method. The bone has a deformation close to 314 zero, which is consistent with its stiffness, and it is clearly

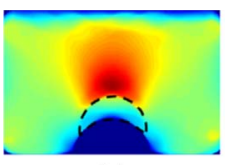

(a)

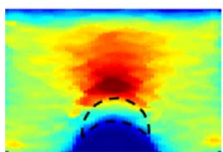

(d)

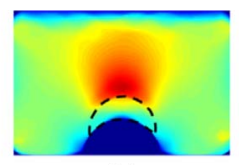

(b)

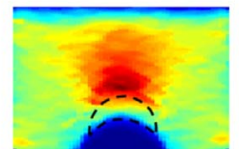

(e)

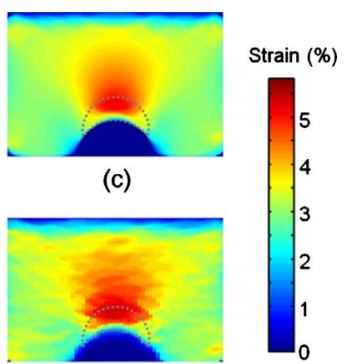

(f)
FIG. 3. Numerical phantom results. On the first row are the theoretical axial strain fields (given by the FEM) and on the second row are the estimated axial strain fields (in \%). (a) and (d) are the fields for the configuration including a $120 \mathrm{kPa}$ pressure ulcer. (b) and (e) are the fields for the configuration with a $90 \mathrm{kPa}$ pressure ulcer. (c) and (f) are the fields for the configuration without pressure ulcer.

identified in the three elastograms. Its position also matches 315 the hyperechoic region of the B-mode image. Although the 316 pressure ulcer cannot be detected in the B-mode scan, the 317 presence of a hard pathological area is revealed in the elas- 318 tograms [Figs. 3(d) and 3(e)]: Strain for the pathological area 319 is indeed half that of its upper neighborhood (about $2.5 \%$ vs 320 $5 \%$ ). Transitions from the healthy tissues to the ulcer and 321 from the ulcer to the bone are very sharp. This underlines the $\mathbf{3 2 2}$ hard region, with limits corresponding to the dotted line 323 (area available from the FEM and added manually on the 324 different maps). In contrast, the maps corresponding to the $\mathbf{3 2 5}$ healthy model [Figs. 3(c) and 3(f)] show a relatively differ- 326 ent configuration, with a smaller maximum and the region of 327 highest strain overlapping the area of the ulcer.

328

\section{PRESSURE ULCER MIMICKING PHANTOM}

In this section, additional tests are reported on a physical 330 phantom designed to mimic an early stage pressure ulcer. A 331 sketch of the phantom is shown in Fig. 4(a). It consisted of a 332 $30 \times 60 \times 110 \mathrm{~mm}^{3}$ parallelepiped, within which three re- 333 gions can be identified: A bone, a region representing the 334 in-development pressure ulcer, and the surrounding healthy 335 tissues. The bone, of $10 \mathrm{~mm}$ in diameter, was a sample from 336 the forward limb of a dog. Other regions were made of poly- 337 vinyl alcohol (PVA) cryogel. PVA cryogel is a polymer that 338 was introduced in the 1990s to build phantoms mimicking 339 soft tissues. ${ }^{37}$ Mixed with Sigmacell particles (Sigmacell 340 Cellulose, type 20, Sigma-Aldrich, USA), its acoustical 341 properties are close to those of soft biological tissues, and 342 this material is suitable for US imaging. Moreover, the ma- 343 terial stiffness increases with the number of freeze-thaw 344 cycles applied. By varying this number of cycles, a wide 345 range of elasticity values can be obtained, especially those 346 commonly met for soft biological tissues. ${ }^{38,39}$ Since a pres- 347 sure ulcer is stiffer than healthy tissues, the PVA cryogel for 348 this region went through two freeze-thaw cycles, while the 349 PVA cryogel for the healthy region underwent a single cycle. 350 Such a pressure ulcer corresponds to a deep tissue injury. 351

RF US data were acquired with an Ultrasonix Sonix RP 352 device (Ultrasonix Medical Corporation, Richmond, BC, 353 Canada), equipped with a $7 \mathrm{MHz}$ linear probe (reference 354 


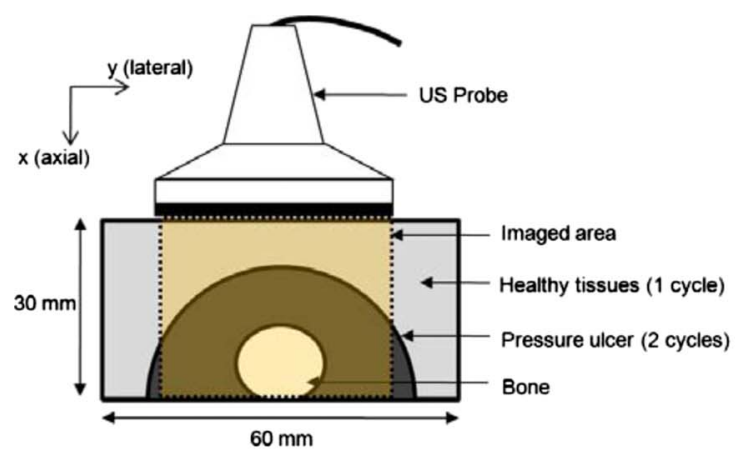

(a)

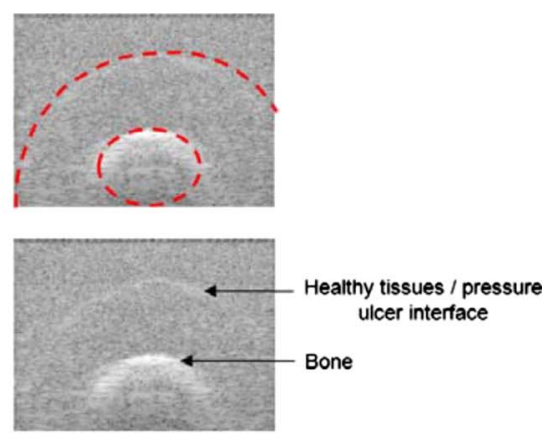

(b)

FIG. 4. (a) PVA phantom constitution. (b) B-mode image of the phantom.

355 L14-5/38). The postcompression data were acquired after 356 uniform compression of the top surface of the phantom (ap357 plied with a plate, while the bottom surface remained fixed). 358 These data were then processed with our strain estimator.

359 As it can be seen from the B-mode image of this region 360 [Fig. 4(b)], the bone could be clearly identified from its 361 specular reflection. However, the echography did not provide 362 any information about the mechanical properties of the phan363 tom. On the contrary, the estimated axial strain map (Fig. 5) 364 was helpful for the detection of the three regions of the phan365 tom. Even if strain values remained within a small range, it 366 can be seen that pathological tissues exhibit a different pat367 tern compared to the surrounding healthy soft tissues. Fi368 nally, deformation of the bone is almost negligible, as ex369 pected.

370 Mean strain were calculated for the different tissue types. 371 They were equal to $1.01 \pm 0.24 \%, 0.45 \pm 0.16 \%$, and $3720.06 \pm 0.06 \%$ for the healthy, pathological, and bone regions, 373 respectively.

\section{V. PRELIMINARY IN VIVO STUDY}

375 To further evaluate the contribution of elastography for 376 pressure ulcer detection, in vivo acquisitions on an experi377 mental rat model were performed. The intent of this study 378 was to investigate in vivo the ability of elastography to detect 379 strain, when the tissues under examination were subjected to 380 a long-term unrelieved pressure.

381 The main reason for tissue breakdown is ischemia, ${ }^{11-13}$ in 382 connection with the interface pressure at the skin level. Tra383 ditionally, the value of $32 \mathrm{~mm} \mathrm{Hg}(4.3 \mathrm{kPa})$ is quoted for the 384 pressure threshold that triggers capillary closure: ${ }^{40}$ If main385 tained, interface pressures higher than this value are sup-
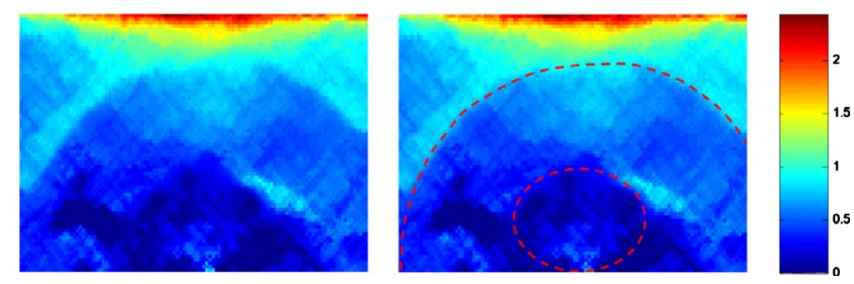

FIG. 5. Estimated strain map (in \%) for the PVA cryogel phantom. posed to lead to tissue damage. However, a more recent 386 study demonstrates that this value is not relevant since cap- 387 illary closure mainly depends on the local pressure. ${ }^{41}$ To in- 388 duce tissue breakdown, we therefore chose to adapt the pro- 389 tocol described by Gefen et al., ${ }^{14}$ where a maintained 390 compression was applied on the thigh of rats to generate 391 pressure ulcers. In the latter paper, it is shown that a pressure 392 of $35 \mathrm{kPa}$ maintained during 60 min leads to a twofold stiff- 393 ening of tissues with cell death.

394

The protocol for animal experimentation was approved by 395 the Animal Care Committee of the Centre Hospitalier de 396 l'Université de Montréal, in accordance with the guidelines 397 of the Canadian Council of Animal Care. The animal was an 398 18-week-old male rat from the Brown Norway breed and 399 weighed $265 \mathrm{~g}$. It was first anesthetized by inhalation of 400 $2.5 \%$ isofluorane, the hair of the hind limb was carefully 401 shaved, and the animal was then immobilized. Its body tem- 402 perature was monitored with a rectal probe (Thermalert 403 TH-5, Physitemp Instruments, Clifton, USA) and maintained 404 at $37 \pm 1{ }^{\circ} \mathrm{C}$ with a heating surface. A first set of RF US data 405

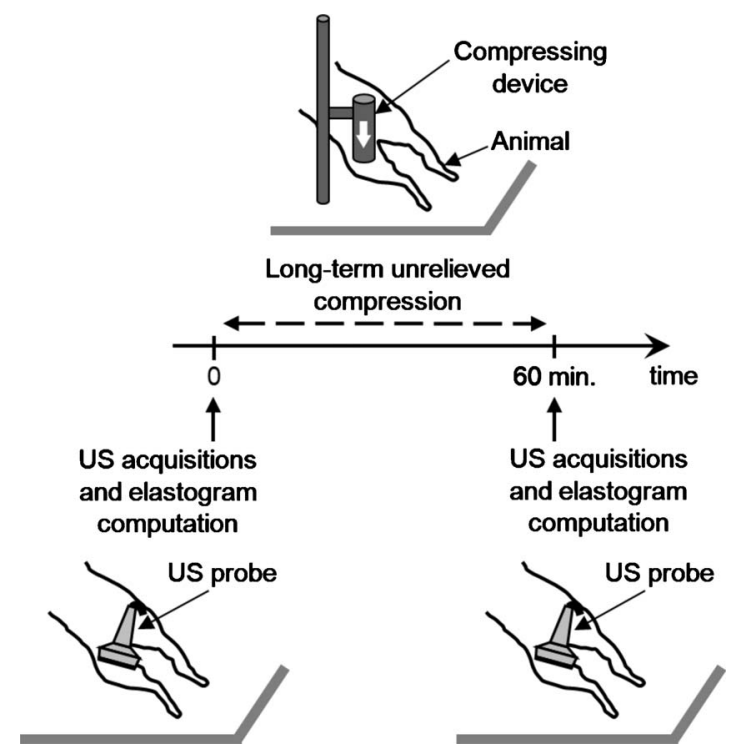

FIG. 6. Scheme of the in vivo protocol used to induce a pressure ulcer early detection on a rat and to acquire US data for elastography imaging. 


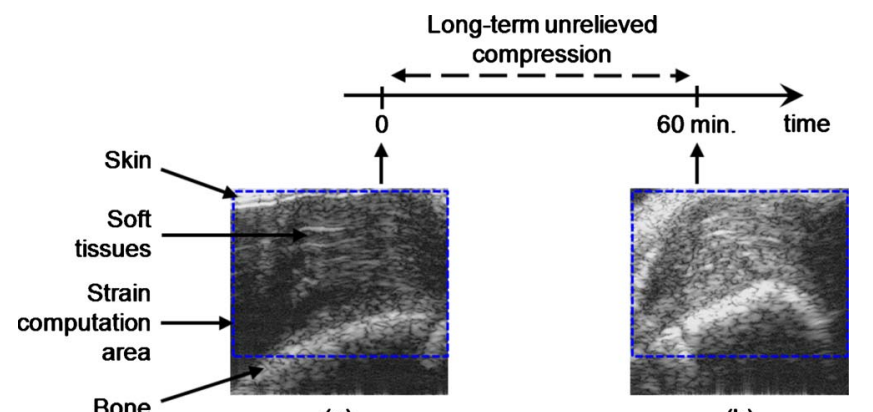

(a)

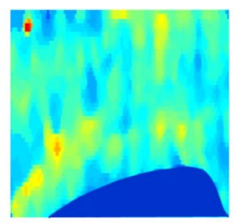

(c)

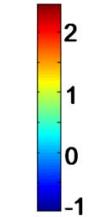

Strain (\%)

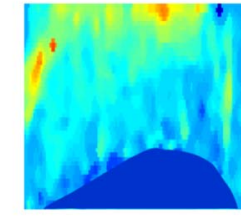

(d)

FIG. 7. B-mode images and elastograms for the in vivo experiment. (a) Initial B-mode image (showing the different tissues). (b) B-mode image after 60 min compression. (c) Initial elastogram. (d) Elastogram after $60 \mathrm{~min}$ compression.

406 was acquired, in a view to compute elastograms. A $35 \mathrm{kPa}$ 407 pressure was then applied on the thigh of the animal during $40860 \mathrm{~min}$ to generate a pressure ulcer. A second set of RF US 409 data was acquired after the $60 \mathrm{~min}$ compression. Once the 410 experiments were completed, the animal was euthanized by 411 inhalation of $\mathrm{CO}_{2}$. Figure 6 summarizes the protocol.

412 RF US data were acquired with a VisualSonics Vevo 660 413 device (VisualSonics Inc., Toronto, Canada), equipped with a $41435 \mathrm{MHz}$ probe dedicated to small animal studies. US data 415 were acquired with the same settings of the scanner and were 416 processed to compute elastograms before the application of 417 the compression and after the 60 min compression. The com418 pression tool was a wooden stick, whose end was a 2-cm-in419 diameter disk in contact with the skin of the animal. Both the 420 US probe and the compression device were mounted on a 421 robotic arm (described in detail in Ref. 42). The latter was a 4226 degrees of freedom industrial robot (F3 Articulated Robot, 423 CRS Robotics Corporation, Burlington, Canada), equipped 424 with a force sensor on its end (ATI, Industrial Automation, 425 Apex, USA), and controlled through a software interface (In426 tegral Technologies Inc., Laval, Canada). This enabled the 427 control of the pressure applied with the compressing device 428 and ensured an accurate positioning of the US probe.

429 Results of this experiment are presented in Fig. 7. Figure 4307 (a) provides a B-mode image of the investigated area where

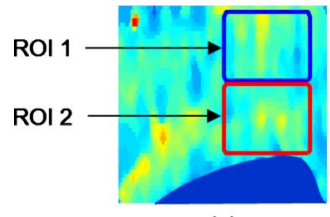

(a)

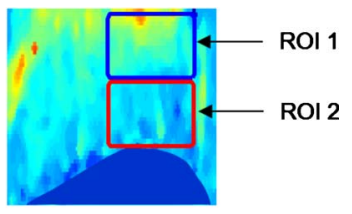

(b)

FIG. 8. Location of the ROIs for mean strain computation (a) on the initial elastogram and (b) on the final elastogram.

TABLE I. Mean strain and standard deviation, according to time and location of the ROI, for the in vivo experiment.

\begin{tabular}{lrrr}
\hline \hline \multirow{2}{*}{$\begin{array}{c}\text { Mean strain } \\
(\%)\end{array}$} & 0 min & $60 \mathrm{~min}$ \\
\cline { 3 - 4 } & Close to the skin & $0.48 \pm 0.22$ & $0.50 \pm 0.27$ \\
ROI location & Close to the bone & $0.47 \pm 0.19$ & $0.13 \pm 0.13$ \\
\hline \hline
\end{tabular}

different tissues can be identified: The skin, the bone (both 431 hyperechoic), and soft tissues, which is the region that was 432 specifically analyzed. The corresponding B-mode image af- 433 ter the application of the 60 min compression is shown in 434 Fig. 7(b). Due to the 60 min compression, the B-mode im- 435 ages exhibit different patterns. However, these images alone 436 are not relevant to reveal or not the presence of a pathologi- 437 cal area. Computed elastograms are displayed in Figs. 7(c) 438 and 7(d), respectively. The initial elastogram appears homo- 439 geneous. Only the area immediately above the bone exhibits 440 a slightly higher strain. As a comparison, the same area on 441 the second elastogram (i.e., after 60 min compression) be- 442 haved differently, with a deformation smaller than the sur- 443 rounding tissues. A sharp evolution of the strain along depth 444 is also visible for the whole image: Shallow tissues seem to 445 exhibit higher strains compared to the tissues near the bone. 446 This evolution tends to show that the tissue area over the 447 bone became stiffer, which could be the indication of an 448 emerging pressure ulcer.

449

To support this visual impression, mean deformations 450 were calculated in two ROIs, as shown in Fig. 8. For a given 451 image, both ROIs have the same size. They are also located 452 at the same lateral position, but at different depths, either 453 near the skin or near the bone. Mean strain was estimated 454 over the pixels located within the perimeter defined by the 455 ROI. The corresponding results are given in Table I. Before 456 the compression was applied $(t=0)$, the strain was almost the 457 same for both regions $(0.48 \%$ for the top ROI and $0.47 \%$ for 458 the bottom ROI). On the other hand, mean strains were sig- 459 nificantly different after the compression of $60 \mathrm{~min}$ : While 460 the mean strain for the top ROI was $0.50 \%$, that for the 461 bottom ROI dropped to $0.13 \%$. The large decrease in strain 462 near the bone might be due to tissue stiffening in this region. 463 This observation allows to assume that the area near the bone 464 was pathological, corresponding to the presence of a pressure 465 ulcer or, at least, corresponding to tissues that underwent a 466 degradation such that they were significantly stiffer than 467 healthy tissues.

468

\section{DISCUSSION AND CONCLUSION}

In this paper, we proposed a new approach based on ul- 470 trasound imaging to address the challenging problem of pres- 471 sure ulcer early detection. Even though US elastography usu- 472 ally finds its main application in cancer tumor detection, we 473 think it has great potential for pressure ulcer early detection. 474 Since this pathology is associated with a stiffening of dam- 475 aged tissues, highlighting abnormally hard areas could in- 476 deed be fundamental. Moreover, deep pressure ulcers are 477 
478 characterized by a deep-to-superficial pattern: The ulcer de479 velops in deep muscle tissues and progresses toward the 480 skin. US elastography is therefore an interesting tool for 481 early detection, when the deep ulcer is still imperceptible by 482 visual inspection.

483 To investigate the usefulness of US elastography, different 484 types of data were processed. Numerical simulations demon485 strated that a pressure ulcer can theoretically be detected at a 486 very early stage with US elastography. Even when the ulcer 487 region was characterized by a low stiffening (ratio of 1.8 488 relative to normal tissues), the estimated elastogram clearly 489 underlined the pathological area.

490 This observation was confirmed by the results obtained on 491 a physical phantom mimicking a pressure ulcer at an early 492 stage. Computed elastograms showed strain differences be493 tween areas mimicking healthy and pathological tissues.

494 Compared to the results on numerical data, these elasto495 grams showed a different strain pattern, which could be at496 tributed to the different designs of the simulated and experi497 mental phantoms. Indeed, for building procedure reasons, the 498 area mimicking the pressure ulcer in the physical phantom is 499 much wider than those in simulations and completely covers 500 the bone. However, both numerical and experimental results 501 demonstrate that US elastography can detect an abnormally 502 stiff area corresponding to a deep pressure ulcer in develop503 ment, even though no particular symptom is visible on the 504 skin.

505 Finally, in vivo experiments were conducted on a rat 506 model. A maintained pressure was applied on the animal 507 thigh during $60 \mathrm{~min}$, with a view to generate a pressure ulcer. 508 It has been shown in a previous study, ${ }^{14}$ based on histologies, 509 that the protocol used in our experiment (in particular, the 510 same compressing device and level of pressure were consid511 ered) leads to irreversible muscle cell death after only $15 \mathrm{~min}$ 512 of exposition. The same paper concludes that changes in me513 chanical properties follow morphological damages and cel514 lular death. RF US data, acquired before and after applica515 tion of this pressure, were processed with our strain 516 estimator. Elastograms revealed a difference in the way tis517 sues behaved before and after the pressure was applied, 518 which strongly suggest the presence of a pathological area.

519 These first results naturally require confirmation by per520 forming many other in vivo experiments. In particular, acqui521 sitions on human patients can be envisaged using lower fre522 quency probe to image a larger area of tissue.

523 Nevertheless, these first experiments demonstrate that US 524 elastography is a promising technique for pressure ulcer de525 tection, especially at an early stage of the pathology, when 526 the disease is still visually undetectable. In the absence of 527 any other technique, this is also a first step toward the devel528 opment of an objective and reliable tool.

$529 \quad{ }^{a)}$ Electronic mail: jean-francois.deprez@eng.ox.ac.uk

$530{ }^{1}$ National Pressure Ulcer Advisory Panel, "Pressure ulcers in America: 531 Prevalence, incidence, and implications for the future. An executive sum532 mary of the National Pressure Ulcer Advisory Panel monograph," Adv. 533 Skin Wound Care 14(4), 208-215 (2001).

$534{ }^{2} \mathrm{~K}$. O'Dea, "Prevalence of pressure damage in hospital patients in the 535 UK," J. Wound Care 2, 221-225 (1993).
${ }^{3}$ S. E. Sedory Holzer, A. Camerota, L. Martens, T. Cuerdon, J. Crystal- 536 Peters, and M. Zagari, "Costs and duration of care for lower extremity 537 ulcers in patients with diabetes," Clin. Ther. 20(1), 169-181 (1998). 538 ${ }^{4} \mathrm{H}$. Miller and J. Delozier, "Cost implications of the pressure ulcer treat- 539 ment guideline," Columbia, MD, Center for Health Policy Studies, 540 1994:17, Contract 2282-91-0070 (1994). 541 ${ }^{5}$ I. Cho and M. Noh, "Braden scale: Evaluation of clinical usefulness in an 542 intensive care unit," J. Adv. Nurs. 66(2), 293-302 (2010). 543 ${ }^{6}$ R. J. Goldman and R. Salcido, "More than one way to measure a wound: 544 An overview of tools and techniques," Adv. Skin Wound Care 15, 236- 545 243 (2002)

546

${ }^{7}$ A. A. Weber, C. Gehin, G. Moddy, J. Jossinet, and E. T. McAdams, 547 "Characterization of a multi-frequency wound impedance mapping instru- $\mathbf{5 4 8}$ ment," Proceedings of the 30th IEEE EMBS Conference, Vancouver, 549 Canada, 20-24 August, 2008.

550

${ }^{8}$ S. Treuillet, B. Albouy, and Y. Lucas, "Three-dimensional assessment of 551 skin wounds using standard digital camera," IEEE Trans. Med. Imaging 552 28(5), 752-762 (2009).

${ }^{9}$ E. S. Andersen and T. Karlsmark, "Evaluation of four non-invasive meth- 554 ods for examination and characterization of pressure ulcers," Skin Res. 555 Technol. 14, 270-276 (2008).

556

${ }^{10}$ R. Meffre, C. Gehin, P. M. Schmitt, F. De Oliveira, and A. Dittmar, "New 557 methodology for preventing pressure ulcers using actimetry and autono- 558 mous nervous system recording," Proceedings of the 28th IEEE EMBS 559 Conference, New York, NY, 30 August-3 September 2006 (unpublished). 560 ${ }^{11}$ R. K. Daniel, D. L. Priest, and D. C. Weatley, "Etiological factors in 561 pressure sores: An experimental model," Arch. Phys. Med. Rehabil. 62, 562 492-498 (1981).

563

${ }^{12}$ S. M. Dinsdale, "Decubitus ulcers: Role of pressure and friction in cau- 564 sation," Arch. Phys. Med. Rehabil. 55, 147-152 (1974). 565

${ }^{13}$ M. Kosiak, "Etiology of decubitus ulcers," Arch. Phys. Med. Rehabil. 42, 566 19-29 (1961).

567

${ }^{14}$ A. Gefen, N. Gefen, E. Linder-Ganz, and S. S. Margulies, "In vivo 568 muscle stiffening under bone compression promotes deep pressure sores," 569 J. Biomech. Eng. 127, 512-524 (2005). 570

${ }^{15}$ E. Linder-Ganz and A. Gefen, "Stress analyses coupled with damage laws $\mathbf{5 7 1}$ to determine biomechanical risk factors for deep tissue injury during sit- $\mathbf{5 7 2}$ ting," J. Biomech. Eng. 131(1), 011003 (2009).

573

${ }^{16}$ C. V. Bouten, C. W. Oomens, F. P. Baaijens, and D. L. Bader, "The 574 etiology of pressure ulcer: Skin deep or muscle bound?," Arch. Phys. 575 Med. Rehabil. 84, 616-619 (2003). 576

${ }^{17}$ C. W. J. Oomens, S. Loerakker, and D. L. Bader, "The importance of 577 internal strain as opposed to interface pressure in the prevention of pres- $\mathbf{5 7 8}$ sure related deep tissue injury," J. Tissue Viability 19(2), 35-42 (2010). 579 ${ }^{18}$ T. A. Krouskop, D. R. Dougherty, and S. F. Levinson, "A pulsed Doppler 580 ultrasonic system for making noninvasive measurements of the mechani- $\mathbf{5 8 1}$ cal properties of soft tissue," J. Rehabil. Res. Dev. 24, 1-8 (1987). 582

${ }^{19}$ J. Ophir, I. Céspedes, H. Ponnekanti, Y. Yazdi, and X. Li, "Elastography: 583 A quantitative method for imaging the elasticity of biological tissues," 584 Ultrason. Imaging 13, 111-134 (1991). 585

${ }^{20}$ M. Tanter, J. Bercoff, A. Athanasiou, T. Deffieux, J. L. Gennisson, G. 586 Montaldo, M. Muller, A. Tardivon, and M. Fink, "Quantitative assess- 587 ment of breast lesion viscoelasticity: Initial clinical results using super- $\mathbf{5 8 8}$ sonic shear imaging," Ultrasound Med. Biol. 34, 1373-1386 (2008). 589 ${ }^{21}$ K. Konig, U. Scheipers, A. Pesavento, A. Lorenz, H. Ermert, and T. 590 Senge, "Initial experiences with real-time elastography guided biopsies of 591 the prostate," J. Urol. (Paris) 174, 115-117 (2005). 592

${ }^{22}$ L. Sandrin, B. Fourquet, J. M. Hasquenoph, S. Yon, C. Fournier, F. Mal, 593 C. Christidis, M. Ziol, B. Poulet, F. Kazemi, M. Beaugrand, and R. Palau, 594 "Transient elastography: A new noninvasive for assessment of hepatic 595 fibrosis," Ultrasound Med. Biol. 29(12), 1705-1713 (2003). 596

${ }^{23}$ R. L. Maurice, J. Ohayon, Y. Frétigny, M. Bertrand, G. Soulez, and G. 597 Cloutier, "Noninvasive vascular elastography: Theoretical framework," 598 IEEE Trans. Med. Imaging 23(2), 164-180 (2004). 599

${ }^{24}$ S. K. Alam, J. Ophir, and E. Konofagou, "An adaptive strain estimator for 600 elastography," IEEE Trans. Ultrason. Ferroelectr. Freq. Control 45, 461- 601 472 (1998).

602

${ }^{25}$ E. Brusseau, C. Perrey, P. Delachartre, M. Vogt, D. Vray, and H. Ermert, 603 "Axial strain imaging using a local estimation of the scaling factor from 604 RF ultrasound signals," Ultrason. Imaging 22(2), 95-107 (2000). 605

${ }^{26}$ E. Brusseau, J. Kybic, J. F. Deprez, and O. Basset, "2D locally regular- 606 ized tissue strain estimation from radio-frequency ultrasound images: the- 607 oretical developments and results on experimental data," IEEE Trans. 608 
609

. Imaging 27(2), 145-160 (2008).

$610{ }^{27}$ A. P. Sarvazyan, O. V. Rudenko, S. D. Swanson, J. B. Fowlkes, and S. Y.

611 Emelianov, "Shear wave elasticity imaging-A new ultrasonic technol-

612 ogy of medical diagnostic," Ultrasound Med. Biol. 24, 1419-1435 (1998).

$613{ }^{28}$ K. Nightingale, M. S. Soo, R. Nightingale, and G. Trahey, "Acoustic

614 radiation force impulse imaging: In vivo demonstration of clinical feasi-

615 bility," Ultrasound Med. Biol. 28, 227-235 (2002).

$616{ }^{29}$ J. Bercoff, M. Tanter, and M. Fink, "Supersonic shear imaging: A new

617 technique for soft tissue elasticity mapping," IEEE Trans. Ultrason. Fer-

618 roelectr. Freq. Control 51(4), 396-409 (2004).

$619{ }^{30}$ J. F. Deprez, E. Brusseau, C. Schmitt, G. Cloutier, and O. Basset, "3D estimation of soft biological tissue deformation from radio-frequency ultrasound volume acquisitions," Med. Image Anal. 13, 116-127 (2009).

${ }^{31}$ P. T. Boggs and J. W. Tolle, "Sequential quadratic programming," Acta Numerica 4, 1-51 (1995).

${ }^{32} \mathrm{~B}$. Barrois, L. Heitler, and P. Ribinik, L'escarre: Les basiques (Edition Asymptote, Paris, 1999).

${ }^{33}$ J. Y. Rho, R. B. Ashman, and C. H. Turner, "Young's modulus of trabecular and cortical bone material: Ultrasonic and microtensile measurements," J. Biomech. 26(2), 111-119 (1993).

${ }^{34}$ B. K. Hoffmeister, S. R. Smith, S. M. Handley, and J. Y. Rho, "Anisotropy of Young's modulus of human tibial cortical bone," Med. Biol. Eng. Comput. 38(3), 333-338 (2000).
"Elastic moduli of breast and prostate tissues under compression," Ultra- 633 son. Imaging 20, 260-274 (1998).

${ }^{36}$ J. Meunier and M. Bertrand, "Ultrasonic texture motion analysis: Theory 635 and simulation," IEEE Trans. Med. Imaging 14(2), 293-300 (1995). 636

${ }^{37}$ K. C. Chu and B. K. Rutt, "Polyvinyl alcohol cryogel: An ideal phantom 637 material for MR studies of arterial flow and elasticty," Magn. Reson. 638 Med. 37(2), 314-319 (1997).

${ }^{38}$ J. Fromageau, E. Brusseau, D. Vray, G. Gimenez, and P. Delachartre, 640 "Characterization of PVA cryogel for intravascular ultrasound elasticity 641 imaging," IEEE Trans. Ultrason. Ferroelectr. Freq. Control 50(10), 1318- 642 1324 (2003).

643

${ }^{39}$ J. Fromageau, J. L. Genisson, C. Schmitt, R. L. Maurice, R. Mongrain, 644 and G. Cloutier, "Estimation of polyvinyl alcohol cryogel mechanical 645 properties with four ultrasound elastography methods and comparison 646 with gold standard testings," IEEE Trans. Ultrason. Ferroelectr. Freq. 647 Control 54(3), 498-509 (2007).

648

${ }^{40} \mathrm{E}$. M. Landis, "Micro-injection studies of capillary blood pressure in hu- 649 man skin," Heart 15, 209-228 (1930).

650

${ }^{41}$ D. L. Bader, "The recovery characteristics of soft tissues following re- 651 peated loading," J. Rehabil. Res. Dev. 27, 141-150 (1990). 652

${ }^{42}$ M. A. Janvier, L. G. Durand, M. H. Roy Cardinal, I. Renaud, B. Chayer, 653 P. Bigras, J. de Guise, G. Soulez, and G. Cloutier, "Performance evalua- 654 tion of a medical robotic 3D-ultrasound imaging system," Med. Image 655 Anal. 12, 275-290 (2008). 